\title{
Suppression of opportunistic infectious bacteria by lactic acid bacteria and antibacterial drugs
}

\author{
Takuya Nakano, DDS (1,2), Susumu Imai, PhD (2), Takumi Ishikawa, DDS (1,2), \\ Tomohiko Terai, PhD (3), Takekazu Okumura, PhD (3), Nobuhiro Hanada, DDS, PhD \\ (2), and Hiroshi Kawahara, DDS, PhD (1)
}

(1) Department of Dental Anesthesiology, (2) Department of Translational Research, Tsurumi University School of Dental Medicine, Yokohama, and (3) Microbiological Research Department, Yakult Central Institute, Yakult Honsha Co., Ltd., Kunitachi, Japan

Purpose: The objective of this study was to investigate inhibitory effect of lactic acid bacteria and antibiotics on the growth of Streptococcus pneumoniae ATCC 33400.

Materials and Methods: Antibiotic activities of five oral probiotic candidate bacteria including two strains of Lactobacillus crispatus, Lactobacillus fermentum, Lactobacillus gasseri and Streptococcus mitis and several antibiotics against $S$. pneumoniae were investigated using radial diffusion assay and competition assay. The biofilm formation of $S$. pneumoniae in the presence of $50 \%$ saturated ammonium sulfate precipitation fraction from L. gasseri and several concentrations of gentamicin were monitored by real time cell analyzer, xCELLigence.

Results: The growth of $S$. pneumoniae was inhibited by two strains of Lactobacillus crispatus, Lactobacillus fermentum and Lactobacillus gasseri. The saturated ammonium sulfate precipitation fraction $(0-30 \%$ and $30-50 \%$ ) from L. gasseri effectively inhibited growth of $S$. pneumoniae. The biofilm formation of $S$. pneumoniae monitored by real time cell analyzer (xCELLigence) was suppressed by gentamicin in dose dependent manner. Antibacterial substance from $L$. gasseri against $S$. pneumoniae was suggested to be small molecular weight substance.

Conclusion: L. gasseri inhibited the growth of $S$. pneumoniae suggesting that L. gasseri will be a candidate of probiotics.

(Asian Pac J Dent 2019; 19: 17-25.)

Key Words: antibiotics, lactic acid bacteria, opportunistic infection, probiotics, Streptococcus pneumoniae

\section{Introduction}

Opportunistic infections in the perioperative phase have a major impact on the patient's life prognosis and postoperative healing processes [1-3]. In addition, treatment and prevention of opportunistic infections at perioperative period are not easy due to the appearance of multidrug resistant bacteria and biofilm formation of opportunistic bacteria [4]. Antimicrobial drugs are used to treat opportunistic infections at the perioperative period, and even if their blood levels increase, sufficient antimicrobials do not migrate to saliva [5-7].

In recent years, studies on the control of opportunistic infectious bacteria by probiotics have been conducted $[8,9]$, and it has been reported that the lactic acid bacteria (LAB) has antibacterial activity against opportunistic infectious bacteria. [10]. Terai et al. [11] screened probiotic candidates against periodontopathic bacteria from human oral cavity and finally selected several candidate bacteria including Lactobacillus crispatus, Lactobacillus fermentum, Lactobacillus gasseri and Streptococcus mitis. Of these bacteria, strains of Lactobacillus crispatus revealed antibacterial activities against Haemophilus influenzae (H. influenzae) [12] and Streptococcus pneumoniae (S. pneumoniae) [13]. H. influenzae, especially type b form (Hib) is one of causative bacteria involved in meningitis, epiglottitis, and severe sepsis [14,15]. S. pneumoniae is one of pathogens of upper respiratory tract infections in humans [16-18].

Therefore, oral care is regarded as important, and control of pathogens such as opportunistic infectious bacteria as well as cariogenic bacteria and periodontopathic bacteria in the oral cavity by probiotics is expected. This study was designed to investigate the inhibitory effect of combined use of antibiotics and probiotics on the 
growth of S. pneumoniae.

\section{Materials and Methods}

\section{Bacterial strains and culture conditions}

The five oral probiotic candidate bacteria (Lactobacillus crispatus YIT 12319 (L.c 1), Lactobacillus crispatus YIT 12945 (L.c 2), Lactobacillus gasseri YIT 12321 (L.g), Lactobacillus fermentum YIT 12320 (L.f) and Streptococcus mitis YIT 12322 (S.m)) were clinical isolates maintained at Microbiological Research Department, Yakult Central Institute, Yakult Honsha Co., Ltd. and Department of Translational Research, School of Dental Medicine, Tsurumi University. All lactobacillus species were grown in Difco Lactobacilli MRS (MRS) Broth (Becton, Dickinson and Company, Sparks, MD, USA) and S. mitis in Bacto Tryptic Soy (TS) Broth medium (Becton, Dickinson and Company). These bacteria were grown under anaerobic condition of $80 \% \mathrm{~N}_{2}, 10 \% \mathrm{CO}_{2}$, and $10 \% \mathrm{H}_{2}$ at $37^{\circ} \mathrm{C}$ for $18-24$ hours. The culture was centrifuged at $1,710 \times \mathrm{g}$ for 20 minutes to collect cell free culture supernatants (CFCSs) to evaluate antibacterial activities.

S. pneumoniae ATCC 33400, S. pneumoniae ATCC 49619 and H. influenzae ATCC 9795 were purchased from American Type Culture Collection (Manassas, VA, USA). S. pneumoniae GTC 261 and H. influenzae GTC 15014 were purchased from GTC Collection (Gifu University Graduate School of Medicine, Gifu, Japan). All strains of $S$. pneumoniae were grown under anaerobic condition of $80 \% \mathrm{~N}_{2}, 10 \% \mathrm{CO}_{2}$, and $10 \% \mathrm{H}_{2}$ at $37^{\circ} \mathrm{C}$ for 18-24 hours. Two strains of $H$. influenzae were anaerobically incubated in Muller Hinton (MH) Broth medium (Becton, Dickinson and Company) supplemented with $150 \mu \mathrm{L} / \mathrm{mL}$ of NAD ${ }^{+}$(Oriental Yeast Co., Ltd., Tokyo, Japan) (culture medium named as $\mathrm{MHN}$ ) at $37^{\circ} \mathrm{C}$ for 24 hours. The cell suspension was prepared with PBS after centrifuging the culture medium at $1,710 \times g$ for 20 minutes.

\section{Minimal inhibitory concentration (MIC)}

An antibiotic-containing liquid medium $(200 \mu \mathrm{L})$ was placed in the wells on the left end row of 96-well plate, and $100 \mu \mathrm{L}$ of the same medium was placed in all other wells. One hundred $\mu \mathrm{L}$ from the first row on the left end was transferred to the second row with eight multiple micropipettes and mixed 5 times, then $100 \mu \mathrm{L}$ of medium were transferred to right one. This handling was repeated to right end row to make a $1 / 2$ serial dilution series of antibiotics. The bacterial suspension $(10 \mu \mathrm{L})$ was inoculated to all wells and incubated under anaerobic condition for 24 to 48 hours. Turbidity of wells was measured at $540 \mathrm{~nm}$ using Micro Plate Reader to confirm bacterial growth after culturing.

\section{Radial diffusion assay (RDA)}

A series of tests were performed by employing RDA [19] to determine the antibacterial activities of antibiotics against five candidate probiotic oral bacteria, S. pneumoniae, and H. influenzae using two layers of agarose gels. The RDA was also used to investigate the antibacterial activities of the five candidate probiotic oral bacteria against $S$. pneumonia.

In the case of L. crispatus YIT 12319, L. crispatus YIT 12945, L. gasseri YIT 12321, and L. fermentum YIT 12320, A-broth containing $0.055 \mathrm{~g}$ of MRS medium, $0.15 \mathrm{~g}$ of agar, and $10 \mathrm{~mL}$ of distilled water (DW) was autoclaved at $121^{\circ} \mathrm{C}$ for 15 minutes. B-broth containing $1.1 \mathrm{~g}$ of MRS medium, $0.15 \mathrm{~g}$ of agar, and $10 \mathrm{~mL}$ of DW was also autoclaved at $121^{\circ} \mathrm{C}$ for 15 minutes. In the case of $S$. mitis YIT 12322 and $S$. pneumoniae ATCC 33400 , A-broth containing $0.03 \mathrm{~g}$ of TS medium, $0.15 \mathrm{~g}$ of agar and $10 \mathrm{~mL}$ of DW, and B-broth containing $0.6 \mathrm{~g}$ of TS medium, $0.15 \mathrm{~g}$ of agar and $10 \mathrm{~mL}$ of DW were autoclaved at $121^{\circ} \mathrm{C}$ for 15 minutes. A-broth and B-broth 
were autoclaved at $105^{\circ} \mathrm{C}$ for 1 minute, and kept warm at $48^{\circ} \mathrm{C}$ in water bath. In the case of H. influenzae, A-broth contained $21 \mathrm{mg}$ of $\mathrm{MH}$ medium, $1.5 \%$ agarose and $10 \mathrm{~mL}$ of DW supplemented with $\mathrm{NAD}^{+}$, and B-broth contained $42 \mathrm{mg}$ of MH medium, 1.5\% agarose and $10 \mathrm{~mL}$ of DW supplemented with $\mathrm{NAD}^{+}$[12].

The pre-cultured bacterial suspension $(300-500 \mu \mathrm{L})$ was added to A-broth. After dispersing the bacteria by gentle mixing, the mixture was poured into petri dish and allowed to solidify inside a clean bench. A series of wells ( $2 \mathrm{~mm}$ in diameter) was punched in the agar, and $5 \mu \mathrm{L}$ of each sample solution was pipetted into a well. Positive control (bacitracin; $20 \mathrm{Unit} / \mathrm{mL}$ ) and negative control (phosphate buffered saline, PBS) were included on each plate. The plates were placed inside the clean bench for 5 minutes at room temperature to allow the experimental solutions to diffuse. B-broth was poured over the base layer and allowed to solidify inside a clean bench. The plates were then turned over and cultured under anaerobic conditions $\left(80 \% \mathrm{~N}_{2}, 10 \% \mathrm{CO}_{2}\right.$, and $10 \%$ $\mathrm{H}_{2}$ ) at $37^{\circ} \mathrm{C}$ for 24 hours. The diameters of clear zone (bacterial growth inhibition) around the wells were measured with a slide caliper [12,20,21].

\section{Competition assay}

Competition assays were performed to objective the growth suppression effects of four candidate oral probiotic bacteria (L. crispatus YIT 12319, L. crispatus YIT 12945, L. gasseri YIT 12321, and L. fermentum YIT 12320) on three strains of S.pneumoniae ATCC 33400, S.pneumoniae GTC 261, and S.pneumoniae ATCC 49619 using agar plates containing a mixture of MRS and TS mediums at a ratio of 1:4. Each pair of competitive species were inoculated on the plate at the same time in close proximity ( $5 \mathrm{~mm}$ or $6 \mathrm{~mm}$ apart) using $5 \mathrm{~mL}$ of overnight cultures after adjusting the concentrations to an $\mathrm{OD}_{540 \mathrm{~nm}}=0.25$, as mentioned above. The plates were incubated at $37^{\circ} \mathrm{C}$ anaerobically for 24 hours before bacterial growth was inspected and scored.

\section{Co-culture of L. gasseri and S. pneumoniae}

The culture of L. gasseri YIT 12321 and S. pneumoniae ATCC 33400 were centrifuged at 1,710 $\times g$ for 20 minutes, and the cells were suspended in PBS and adjusted to $\mathrm{OD}_{540 \mathrm{~nm}}=0.25$. A bacterial cell suspension was mixed at a ratio of 1:1 in the mixture of TS medium and MRS medium at a ratio of $4: 1$ and cultured at $37^{\circ} \mathrm{C}$ for 0 , 30, 60, and 120 minutes, (and 6 hours in another case) in the presence or absence of an antibacterial agent under anaerobic condition $\left(80 \% \mathrm{~N}_{2}, 10 \% \mathrm{CO}_{2}\right.$, and $\left.10 \% \mathrm{H}_{2}\right)$. An aliquot of bacterial culture was inoculated on the agar medium composed of TS and MRS medium at a ratio of 4:1 using a spiral plating instrument (Eddy Jet, IUL, Barcelona, Spain) and cultured under anaerobic condition at $37^{\circ} \mathrm{C}$ for 48 hours to calculate colony forming unit (CFU).

\section{Real-time monitoring of biofilm formation of $S$. pneumoniae}

A real-time cell analyzer (RTCA), xCELLigence (ACEA Bioscience Inc., San Diego, CA, USA) is an instrument to quantify cell proliferation, morphology change and cell-substrate attachment in a real-time manner. A bottom surface of microtiter plate was covered with gold electrode. The xCELLigence was used to monitor biofilm formation of several bacteria [22]. Biofilm formation of $S$. pneumoniae was monitored by xCELLigence in this study. The xCELLigence was set in the incubator. Bacterial broth medium in the presence of gentamicin $(0-25 \mu \mathrm{g} / \mathrm{mL}), 30 \%$ saturated ammonium sulfate fraction from L. gasseri and bacterial suspension of $S$. pneumoniae were added to each well of E-plate for xCELLigence and cultured at $37^{\circ} \mathrm{C}$ for 40 hours.

\section{Sodium dodecyl sulfate polyacrylamide gel electrophoresis (SDS-PAGE)}

Tricine-SDS PAGE was performed with 15\% acrylamide gel (e-PAGEL, ATTO Corp, Tokyo, Japan). The molecular mass standards and sample solutions were applied on the gel. After electrophoresis, resulting gel was 
cut into two parts. One was stained with EzStain (AE-1340 EzStain Aqua, ATTO Corp.), and the other was incubated with $1 \%$ Triton $\mathrm{X}-100$ for 30 minutes, followed by three times rinsing with sterile water for 30 minutes, and finally rinsed with PBS for 30 minutes. The gel was overlaid on the TS agar plate including $S$. pneumoniae, and cultured under anaerobic condition at $37^{\circ} \mathrm{C}$ for 24 hours.

\section{Results}

\section{MIC of antibiotics against oral probiotic candidates and opportunistic bacteria}

Results of MIC of antibiotics against oral probiotic candidate bacteria are shown in Table 1. The five probiotic candidate bacteria were relatively resistant to antibiotics used. L.c 1 and L.c 2 were resistant to gentamicin and ofloxacin. L. $f$ and L.g were resistant to many antibiotics except for ampicillin and erythromycin, respectively. $S$. $m$ was sensitive to all antibiotics used. The MIC of antibiotics against opportunistic infectious bacteria was relatively low (Table 2). The two strains of $H$. influenzae were sensitive to five kinds of antibiotics except for vancomycin. The two kinds of $S$. pneumoniae were sensitive to all antibiotics used and especially sensitive to ampicillin.

Table 1 MIC of antibiotics against oral probiotic candidate bacteria

\begin{tabular}{ccccccc}
\hline Oral probiotic bacteria & $\begin{array}{c}\text { L. crispatus } \\
(\text { L. } c 1)\end{array}$ & $\begin{array}{c}\text { L. crispatus } \\
(\text { L. } 2)\end{array}$ & $\begin{array}{c}\text { L.fermentum } \\
(\text { L. } f)\end{array}$ & $\begin{array}{c}\text { L.gasseri } \\
(\text { L. } g)\end{array}$ & $\begin{array}{c}\text { S. mitis } \\
(S . m)\end{array}$ \\
\hline & Ampicillin & 0.78 & 1.56 & 0.39 & $200 \leqq$ & 0.0125 \\
& Erythromycin & 3.13 & 1.56 & $200 \leqq$ & 3.13 & 0.39 \\
Antibiotic $(\mu \mathrm{g} / \mathrm{mL})$ & Gentamicin & $200 \leqq$ & $200 \leqq$ & $200 \leqq$ & $200 \leqq$ & 50 \\
& Ofloxacin & $200 \leqq$ & $200 \leqq$ & $200 \leqq$ & $200 \leqq$ & 6.25 \\
& Tetracycline & 100 & 25 & $200 \leqq$ & $200 \leqq$ & 25 \\
& Vancomycin & 1.56 & 12.5 & $200 \leqq$ & $200 \leqq$ & 1.56 \\
\hline
\end{tabular}

Table 2 MIC of antibiotics against opportunistic infectious bacteria

\begin{tabular}{|c|c|c|c|c|c|}
\hline \multicolumn{2}{|c|}{ Opportunistic infection bacteria } & $\begin{array}{l}\text { H. influenzae } \\
\text { ATCC } 9795\end{array}$ & $\begin{array}{c}\text { H. influenzae } \\
\text { GTC } 15014\end{array}$ & $\begin{array}{c}\text { S. pneumoniae } \\
\text { ATCC } 49619\end{array}$ & $\begin{array}{c}\text { S. pneumoniae } \\
\text { ATCC } 33400\end{array}$ \\
\hline \multirow{6}{*}{ Antibiotic $(\mu \mathrm{g} / \mathrm{mL})$} & Ampicillin & 0.2 & 0.2 & 0.0125 & 0.025 \\
\hline & Erythromycin & 1.56 & 1.56 & 0.1 & 0.05 \\
\hline & Gentamicin & 3.13 & 3.13 & 6.25 & 12.5 \\
\hline & Ofloxacin & 0.025 & 0.025 & 0.78 & 1.56 \\
\hline & Tetracycline & 0.39 & 0.39 & 0.78 & 0.2 \\
\hline & Vancomycin & $200 \leqq$ & $200 \leqq$ & 0.78 & 0.78 \\
\hline
\end{tabular}

\section{Minimum concentration of antibiotics to reveal clear zone on agar medium}

The antibacterial activities of antibacterial substances were often determined by RDA [19]. Therefore, minimum concentration of antibiotics to reveal clear zone on the agar medium against probiotic candidate bacteria was examined by RDA. The results were shown in Table 3. Four lactobacilli were resistant to ofloxacin. Other antibiotics showed variety of antibacterial activity against LAB used. Generally minimum concentration of antibiotics to reveal clear zone assessed by RDA were higher than that of MIC in Table 1 . The minimum concentrations of antibiotics to reveal clear zone assessed by RDA against opportunistic infectious bacteria were shown in Table 4. The minimum concentration of antibiotics assessed by RDA was higher than that of MIC in Table 2. 
Table 3 Minimum concentration of antibiotics to reveal clear zone assessed by RDA against probiotic candidate bacteria

\begin{tabular}{clccccc}
\hline \multicolumn{2}{c}{ Oral probiotic bacteria } & $L . c 1$ & $L . c 2$ & $L . f$ & $L . g$ & $S . m$ \\
\hline & Ampicillin & 50 & 25 & 6.25 & 12.5 & 1.56 \\
& Erythromycin & 3.13 & 6.25 & 6.25 & 3.13 & 0.78 \\
Antibiotic & Gentamicin & 200 & 200 & 25 & 25 & 200 \\
$(\mu \mathrm{g} / \mathrm{mL})$ & Ofloxacin & $800 \leqq$ & $800 \leqq$ & $800 \leqq$ & $800 \leqq$ & 25 \\
& Tetracycline & 50 & 50 & 200 & 100 & 12.5 \\
& Vancomycin & $800 \leqq$ & 25 & $800 \leqq$ & 25 & 1.56 \\
\hline
\end{tabular}

Table 4 Minimum concentration of antibiotics to reveal clear zone assessed by RDA against opportunistic infectious bacteria

\begin{tabular}{cccc}
\hline \multicolumn{2}{c}{ Opportunic infection bacteria } & $\begin{array}{c}\text { H. influenzae } \\
\text { ATCC 9795 }\end{array}$ & $\begin{array}{c}\text { S. pneumoniae } \\
\text { ATCC 33400 }\end{array}$ \\
\hline \multirow{5}{*}{ Antibiotic $(\mu \mathrm{g} / \mathrm{mL})$} & 6.25 & 1.56 \\
& Ampicillin & 50 & 0.78 \\
& Erythromycin & 12.5 & 25 \\
& Gentamicin & 0.2 & 50 \\
& Ofloxacin & 0.39 & 3.13 \\
& Tetracycline & 400 & 6.25 \\
& Vancomycin & & \\
\hline
\end{tabular}

\section{Competition assay between $L$. gasseri and $S$. pneumoniae}

The competition assay between probiotic candidate Lactobacilli and S. pneumoniae were performed (Fig.1). L.c 2 showed strong growth inhibition against S. pneumoniae, and L.c 1, L.f and L.g showed moderate growth inhibition. L.c 1 was reported to inhibit H. influenzae [12], and L.c 2 was demonstrated to inhibit S. pneumoniae [13]. In this study, therefore, the growth inhibition of S. pneumoniae by L.g was investigated in detail.

\section{RDA of culture supernatant of $L$. gasseri}

The antibacterial activities of culture supernatant of L. gasseri and the fraction obtained with ammonium sulfate precipitation against $S$. pneumoniae were measured by RDA (Fig. 2). The supernatant showed obviously positive antibacterial activity and $0-30 \%$ saturated ammonium sulfate fraction and $30-50 \%$ fraction revealed stronger activities. A 50-80\% saturated ammonium sulfate fraction did not show antibacterial activity (data not shown).

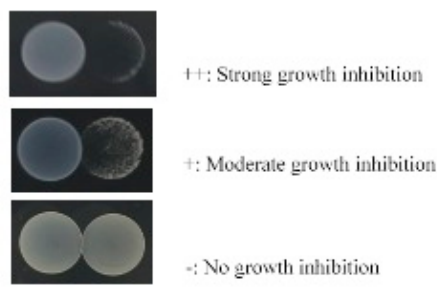

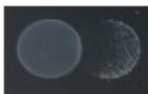

Le I $S p$

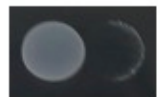

$L C 2 \quad S p$

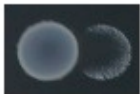

Lf $S p$

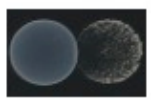

L.g Sp

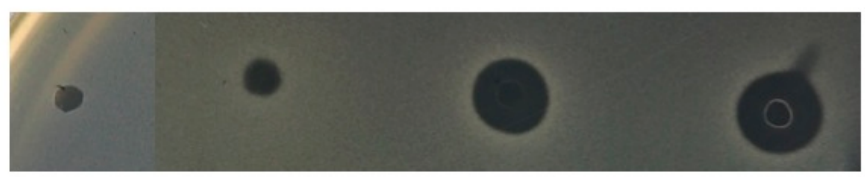

$30 \%$ sat.

$50 \%$ sat.

Fig. 1 (left) Competition assay between Lactobacilli and S. pneumoniae ATCC 33400

Fig. 2 (right) RDA of culture supernatant of L. gasseri. NC: negative control (PBS), Sup: culture supernatant of modified MRS, $30 \%$ sat.: $0-30 \%$ saturated ammonium sulfate fraction, $50 \%$ sat.: $30-50 \%$ saturated ammonium sulfate fraction

\section{Co-culture of L. gasseri and S. pneumoniae}

The co-culture of L. gasseri and S. pneumoniae ATCC 33400 was performed in the presence or absence of gentamicin $(3.13 \mu \mathrm{g} / \mathrm{mL})$. As shown in Figs. 3a and 3b, the cell number of L. gasseri was not changed up to 6 
hours culture. On the other hand, the cell number of $S$. pneumoniae decreased after 2 hours culture, and disappeared after 6 hours culture (Figs $4 \mathrm{a}$ and $4 \mathrm{~b}$ ).
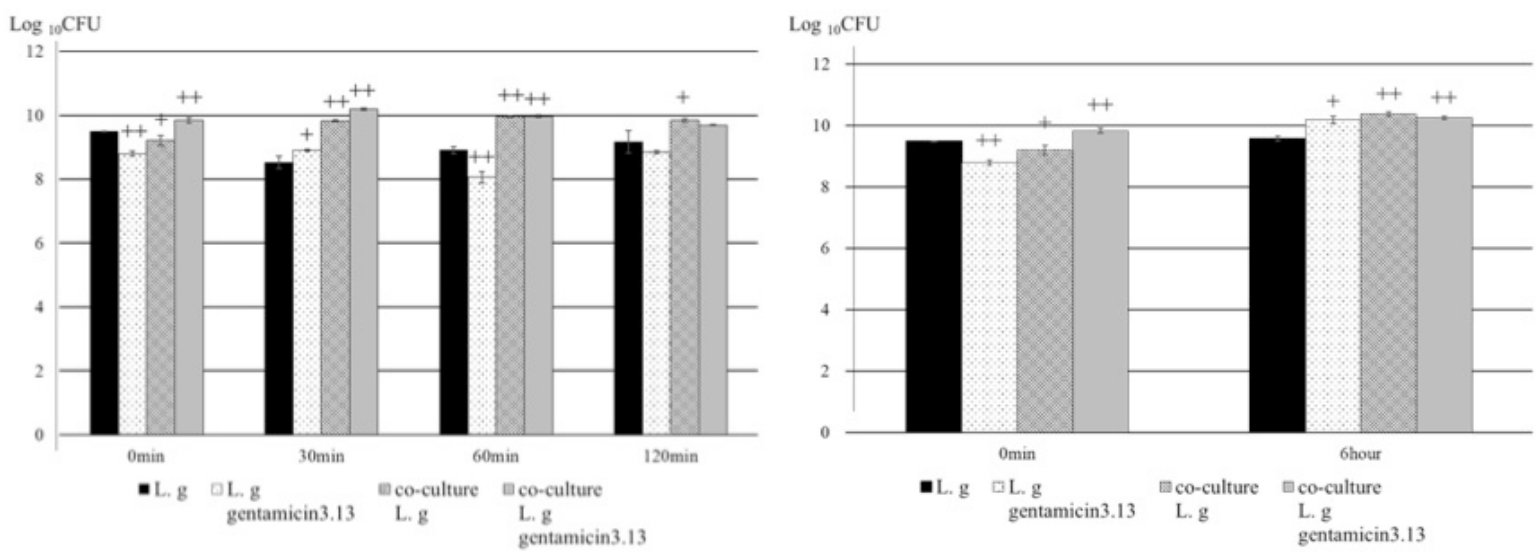

t-test $+p<0.05,++p<0.01$

Fig. 3a (left) CFU of $L . g$ in co-culture of $L$. $g$ and S. $p$ during 2 hours in the presence of GM $3.13 \mu \mathrm{g} / \mathrm{mL}(n=3)$

Fig. 3b (right) CFU of $L$. $g$ in co-culture of $L$. $g$ and $S$. $p$ for 6 hours in the presence of GM $3.13 \mu \mathrm{g} / \mathrm{mL}(n=3)$
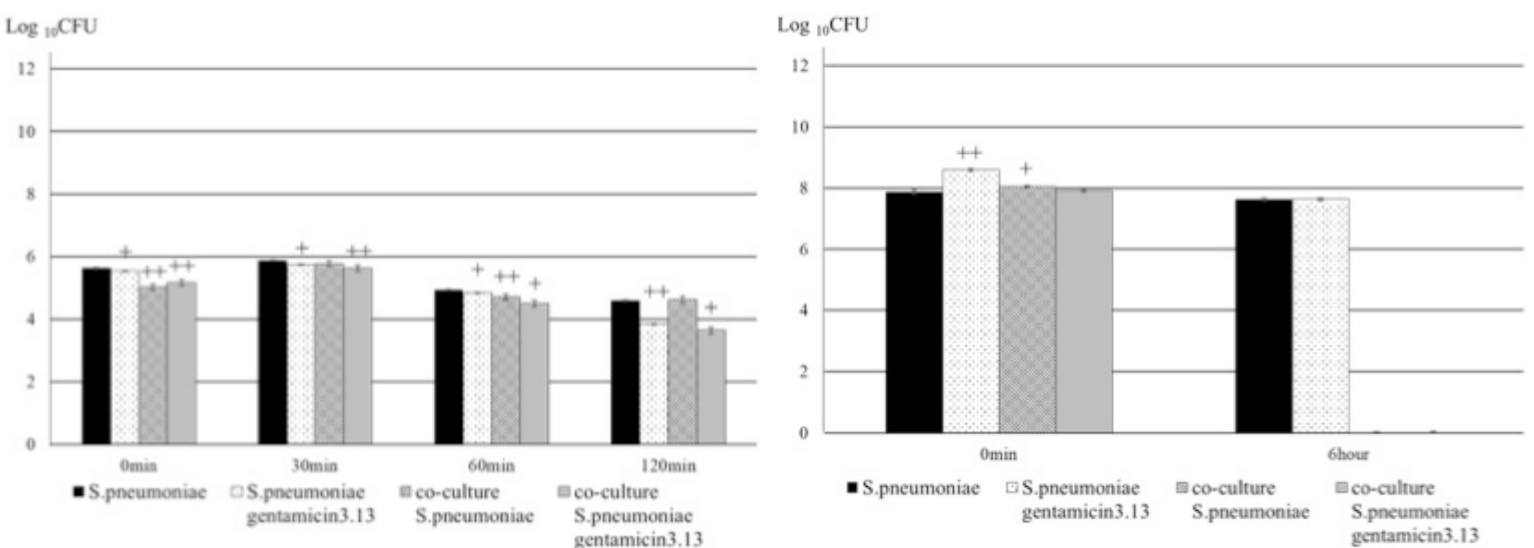

t-test $+p<0.05,++p<0.01$

Fig. 4a (left) CFU of $S$. $p$ in co-culture of $L$. $g$ and $S$. $p$ during 2 hours in the presence of GM $3.13 \mu \mathrm{g} / \mathrm{mL}(n=3)$

Fig. $4 \mathrm{~b}$ (right) CFU of $S$. $p$ in co-culture of $L$. $g$ and S. $p$ for 6 hours in the presence of GM $3.13 \mu \mathrm{g} / \mathrm{mL}(n=3)$.

\section{Real-time monitor of $S$. pneumoniae biofilm formation by xCELLigence}

The biofilm formation of $S$. pneumoniae was monitored by xCELLigence in real-time manner in the presence of $50 \%$ saturated ammonium sulfate fraction from L. gasseri and various concentrations of gentamicin (GM) (Fig. 5). As shown in Table 2, the MIC of gentamicin against $S$. pneumoniae was $12.5 \mu \mathrm{g} / \mathrm{mL}$. The biofilm formation curve in the presence of $6.25 \mu \mathrm{g} / \mathrm{mL}$ gentamicin was slightly inhibited compared to that of control. The biofilm formation curves in the presence of more than MIC $(12.5 \mu \mathrm{g} / \mathrm{mL})$ of gentamicin were inhibited in dose dependent manner.

\section{SDS-PAGE of $50 \%$ saturated ammonium sulfate fraction}

Fig. 6 shows the results of SDS-PAGE and subsequent staining with EzStain (left) and for antibacterial activity (right; clear zone on the gel shown with arrow). Antibacterial activity was observed around molecular mass of 2-3KD. 

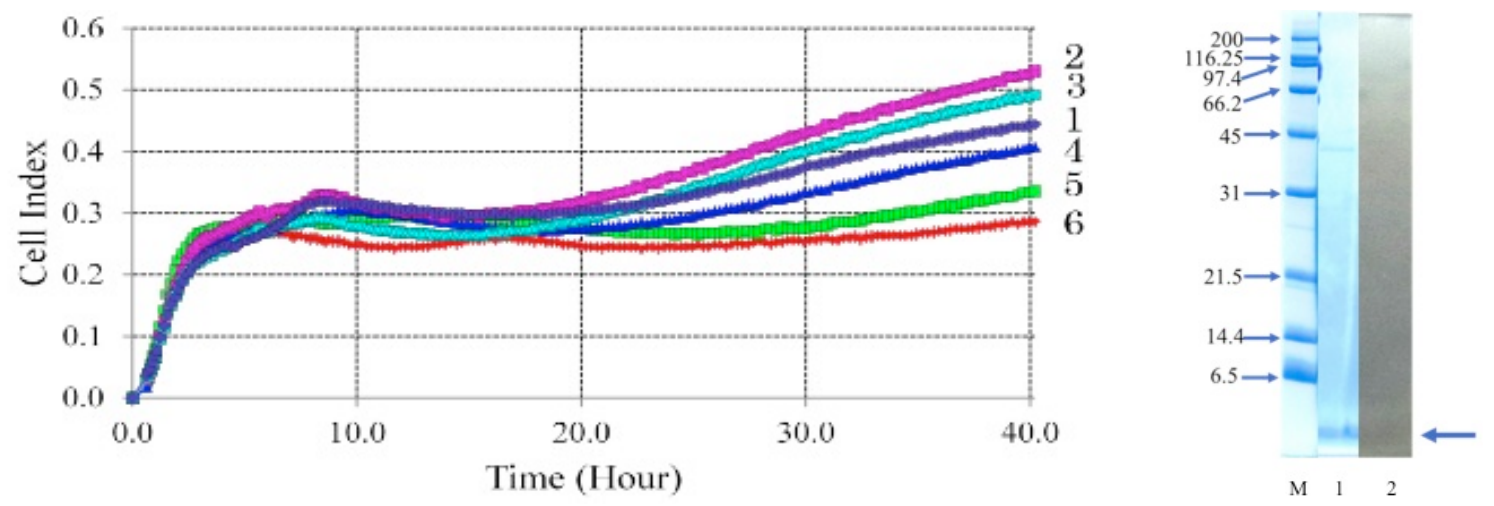

Fig. 5 (left) Real-time monitoring of biofilm formation of S. pneumoniae by xCELLigence in the presence of $50 \%$ saturated ammonium sulfate fraction from $L$. gasseri and various concentrations of gentamicin (GM).

1, GM (0); 2, GM (1.56), 3: GM (3.13), 4: GM (6.25), 5: GM (12.5), 6: GM (25) $(\mu \mathrm{g} / \mathrm{mL})$

Fig. 6 (right) SDS-PAGE analysis of $50 \%$ saturated ammonium sulfate fraction of $L$. gasseri

Lane M: molecular mass standards; 200, 116.25, 97.4, 66.2, 45, 31, 21.5, 14.4, and $6.5 \mathrm{kDa}$, Lane 1: $50 \%$ saturated ammonium sulfate fraction stained with EzStain, Lane 2: gel overlaid on agar medium containing S. pneumoniae

\section{Discussion}

The MIC of six kinds of antibiotics against five strains of probiotic candidate bacteria were relatively high except for S. mitis (Table 1), and that against opportunistic bacteria such as H. influenzae and S. pneumoniae were relatively low (Table 2). These results showed that four strains of Lactobacillus were relatively resistant to antibiotics used and opportunistic bacteria were relatively sensitive to antibiotics. This difference was utilized in this study to examine the effect of co-culture of $L$. gasseri and S. pneumoniae in the presence of antibiotics. The minimum concentration of antibiotics to reveal clear zone on the agar medium against probiotic candidate bacteria and opportunistic bacteria was examined by RDA. Generally minimum concentration of antibiotics to reveal clear zone assessed by RDA was higher than that of MIC. Existence of agar and difference of collision rate between antibiotics and bacteria would influence on the results.

All strains of Lactobacillus used showed antibacterial activity against S. pneumoniae in competition assay (Fig. 1). In this study, the effect of L. gasseri on the growth of S. pneumoniae ATCC 33400 was investigated in detail. The antibacterial activities of $L$. gasseri against $S$. pneumoniae were observed in culture supernatant, $0-30 \%$ and $30-50 \%$ saturated ammonium sulfate precipitation fractions assessed by RDA (Fig. 2). The antibacterial activity of Lactobacillus crispatus LBS 17-11 (same as YIT 12945) against S. pneumoniae ATCC 33400 were reported in $35-80 \%$ saturated ammonium sulfate precipitation fraction [13]. They reported that the antibacterial activity was ascribed to the substance with molecular mass of approximately $7 \mathrm{kDa}$. In this study, the antibacterial substance in $30-50 \%$ saturated ammonium sulfate precipitation fractions was examined by SDS-PAGE, and antibacterial activity against $S$. pneumoniae was observed at a band with molecular mass of 2-3 kDa (Fig. 6). L. gasseri is known to produce some kinds of gassericin such as gassericin A [23], E [24], and T [25]. Molecular mass of these gassericin are around $5 \mathrm{kDa}$. The similarity between these gassericin and our antibacterial substance is unclear. The entity of antibacterial substance in this study is unidentified, so that we need further investigation in the future.

In co-culture experiment, the growth of S.pneumoniae was inhibited by sub-dose of MIC of gentamicin in the presence of L. gasseri (Figs. 4a and 4b). Combined effect of L. gasseri and antibiotics would be suggested to suppress the growth of $S$. pneumoniae. Because limited amounts of gentamicin in blood is secreted into saliva, sufficient concentration of gentamicin will not be expected in saliva [7]. The help by probiotics will promote better condition of oral cavity. 
A real-time cell analyzer (RTCA), xCELLigence is an instrument to quantify cell proliferation, morphology change and cell-substrate attachment in a real-time manner. xCELLigence is used in wide fields including cell biology and bacteriology [26]. In fact, biofilm formation of some bacteria including S. aureus was investigated by using xCELLigence [27]. In this study, xCELLigence was employed to monitor real-time biofilm formation of $S$. pneumoniae in the presence of $50 \%$ saturated ammonium sulfate precipitation fraction and various concentrations of gentamicin, and the biofilm formation was inhibited by gentamicin (same as MIC level and more) in dose dependent manner (Fig. 5). The probiotics will help to maintain healthy oral circumstances and $L$. gasseri might be candidate of oral probiotics for healthy individuals and for especially patients in the prognosis and postoperative healing processes.

\section{Acknowledgments}

This study was supported in part by Research Fund from Yakult Central Institute Tokyo, Japan. We thank staffs of the Department of Dental Anesthesiology and Department of Translational Research, School of Dental Medicine, Tsurumi University for valuable advice and great assistance to complete this study.

\section{Conflict of Interest}

N. Hanada and S. Imai received research fund from Yakult Central Institute (Tokyo, Japan). All remaining authors declare no potential conflicts of interest with respect to authorship and publication of this article.

\section{References}

1. Zawadzki PJ, Perkowski K, Starościak B, Baltaza W, Padzik M, Pionkowski K, et al. Identification of infectious microbiota from oral cavity environment of various population group patients as a preventive approach to human health risk factors. Ann Agric Environ Med 2016; 23: 566-9.

2. Razanajatovo NH, Guillebaud J, Harimanana A, Rajatonirina S, Ratsima EH, Andrianirina ZZ, et al. Epidemiology of severe acute respiratory infections from hospital-based surveillance in Madagascar, November 2010 to July 2013. PLoS One 2018; 13(11): e0205124.

3. Sittitrai P, Srivanitchapoom C, Reunmakkaew D. Deep neck infection in patients with and without human immunodeficiency virus: a comparison of clinical features, complications, and outcomes. Br J Oral Maxillofac Surg 2018; S0266-4356(18)30632-6.

4. El-Sokkary RH, Ramadan RA, El-Shabrawy M, El-Korashi LA, Elhawary A, Embarak S, et al. Community acquired pneumonia among adult patients at an Egyptian university hospital: bacterial etiology, susceptibility profile and evaluation of the response to initial empiric antibiotic therapy. Infect Drug Resist 2018; 11: 2141-50.

5. Ducci M, Scalori V, del Tacca M, Soldani G, Bernardini C, Grothe E, et al. The pharmacokinetics of two erythromycin esters in plasma and in saliva following oral administration in humans. Int J Clin Pharmacol Ther Toxicol 1981; 19: 494-7.

6. Stjernquist-Desatnik A, Samuelsson $P$, Walder $M$. Penetration of penicillin $V$ to tonsillar surface fluid in healthy individuals and in patients with acute tonsillitis. J Laryngol Otol 1993; 107: 309-12.

7. Spencer H, Kozlowska W, Davies JC, Webber P, Chadwick M, Kerr J, et al. Measurement of tobramycin and gentamicin in saliva is not suitable for therapeutic drug monitoring of patients with cystic fibrosis. J Cyst Fibros 2005; 4(3): 209.

8. Rossoni RD, Fuchs BB, de Barros PP, Velloso MD, Jorge AO, Junqueira JC, et al. Lactobacillus paracasei modulates the immune system of Galleria mellonella and protects against Candida albicans infection. PLoS One 2017; 12 (3): e0173332.

9. Jun H, Lingli C, Wenyong Z, Min S, Liu L, Chunlin X, et al. Lactobacillus frumenti facilitates intestinal epithelial barrier function maintenance in early-weaned piglets. Front Microbiol 2018; 9: 1-18.

10. Fahime M, Morteza E, Shabnam R, Davood D, Sarokhalila M, Talebia M, et al. Characterization of bacteriocin production in Lactobacillus spp. isolated from mother's milk. Microbial Pathogenesis 2018; 242-6.

11. Terai T, Okumura T, Imai S, Nakao M, Yamaji K, Ito M, et al. Screening of probiotic candidates in human oral bacteria for the prevention of dental disease. PLoS One 2015; 10(6): e0128657.

12. Sogabe-Ashigaki K, Imai S, Okada A, Matin K, Akimaru G, Terai T, et al. Effects of Lactobacillus crispatus as a candidate for oral probiotic bacteria on Haemophilus influenzae. Asian Pac J Dent 2015; 15: 3-11.

13. Akimaru G, Imai S, Sogabe K, Okada A, Terai T, Okumura T, et al. Characterization and purification of a bacteriocin-like substance produced by Lactobacillus crispatus LBS 17-11 isolated from an oral cavity of human subject. Asian Pac J Dent 2017; 17: 9-14.

14. LiPuma JJ, Richman H, Stull TL. Haemocin, the bacteriocin produced by Haemophilus influenzae: species distribution and role in colonization. Infect Immun 1990; 58: 1600-5.

15. Hallström T, Resman F, Ristovski M, Riesbeck K. Binding of complement regulators to invasive nontypeable Haemophilus influenzae isolates is not increased compared to nasopharyngeal isolates, but serum resistance is linked to disease severity. J Clin Microbiol 2010; 48: 921-7.

16. Santagati M, Scillato M, Patanè F, Aiello C, Stefani S. Bacteriocin-producing oral streptococci and inhibition of respiratory pathogens. FEMS Immunol Med Microbiol 2012; 65: 23-31.

17. Wang J, Liu K, Ariani K, Tao L, Zhang J, Qu JM. Probiotics for preventing ventilator-associated pneumonia: a systematic review and meta-analysis of high-quality randomized controlled trials. PLoS One 2013; 8(12): e83934.

18. Bo L, Li J, Tao T, Bai Y, Ye X, Hotchkiss RS, et al. Probiotics for preventing ventilator-associated pneumonia. Cochrane Database Syst Rev 2014; CD009066.

19. Bosch M, Nart J, Audivert S, Bonachera MA, Alemany AS, Fuentes MC, et al. Isolation and characterization of probiotic strains for improving oral health. Arch Oral Biol 2012; 57: 539-49. 
20. Lehrer RI, Rosenman M, Harwig SS, Jackson R, Eisenhauer P. Ultrasensitive assays for endogenous antimicrobial polypeptides. J Immunol Methods 1991; 137: 167-73.

21. Morita Y, Imai S, Hanyuda A, Matin K, Hanada N, Nakamura Y. Effect of silver ion coating of fixed orthodontic retainers on the growth of oral pathogenic bacteria. Dent Mater J 2014; 33: 268-74.

22. Ferrer MD, Rodriguez JC, Alvarez L, Artacho A, Royo G, Mira A. Effect of antibiotics on biofilm inhibition and induction measured by real-time cell analysis. J Appl Microbiol 2017; 122: 640-50.

23. Pandey N, Malik RK, Kaushik JK, Singroha G. Gassericin A. Circular bacteriocin produced by lactic acid bacteria Lactobacillus gasseri. World J Microbiol Biotechnol 2013; 29: 1977-87.

24. Maldonado B, Caballero-Guerrero B, Martín V, Ruiz-Barba JL, Rodríguez JM. Purification and genetic characterization of gassericin E, a novel co-culture inducible bacteriocin from Lactobacillus gasseri EV1461 isolated from the vagina of a healthy woman. BMC Microbiol 2016; 16(37): 1-13.

25. Birri DJ, Brede DA, Tessema GT, Nes IF. Bacteriocin production, antibiotic susceptibility and prevalence of haemolytic and gelatinase activity in faecal lactic acid bacteria isolated from healthy Ethiopian infants. Microb Ecol 2013; 65: 504-16.

26. Mou X, Wan S, Li Y, Zhang S, Sun M, Liu F, et al. Phenotypic pattern-based assay for dynamically monitoring host cellular responses to Salmonella infections. PLoS One 2011; 6(11): e26544.

27. Kristyna C, Dagmar C, Petr M, Amitava M, Roman G, Pavel K, et al. Staphylococcus aureus and MRSA growth and biofilm formation after treatment with antibiotics and SeNPs. Int J Mol Sci 2015; 24656-24672. 16.

\section{Correspondence to:}

Dr. Susumu Imai

Department of Translational Research, School of Dental Medicine, Tsurumi University

2-1-3, Tsurumi, Tsurumi-ku, Yokohama 230-8501, Japan

Fax: +81-45-573-2473 E-mail: imai-s@tsurumi-u.ac.jp

Copyright (C2019 by the Asian Pacific Journal of Dentistry.

Accepted December 15, 2018. Online ISSN 2185-3487, Print ISSN 2185-3479 